Original Research Article

\title{
The correlation of placental histopathology with neonatal outcome
}

\author{
Kumar S. ${ }^{1}$, Sudarshan $\mathbf{V}^{2}$ \\ ${ }^{1}$ Dr Sunil Kumar, Senior Resident, Department of Pediatrics, Kalpana Chawla Government Medical College, Karnal, \\ Haryana, ${ }^{2}$ Dr. Vijaya Sudarshan, Retd. Head and Prof, Pt. JNM Medical College, Raipur (C.G.), India.
}

Corresponding Author: Dr. Sunil Kumar, Senior Resident, Department of Pediatrics, Kalpana Chawla Government Medical College, Karnal, Haryana. Email: drsunilx@yahoo.com

\begin{abstract}
Introduction: Placenta is a vital organ for the wellbeing of the fetus. Neonatal morbidity and mortality may have its root in the placenta which can be studied from its pathological examination. Objective: This study was designed to explore the correlation of placental histopathology with neonatal outcome. Methods: The study was conducted at the Department of Obstetrics and Gynecology Pediatrics, Raipur. In the study, 100 placentas (with their membrane and cords) were included, among which 76 placentas belonging to neonates with different morbidities like preterm delivery, birth asphyxia, IUGR, septicemia were studied for histopathological changes. Results: Preterm neonates were found to have more infective and inflammatory lesions in the form of chorioamnionitis (33\%). In our study, a higher incidence of villitis was found in IUGR neonates (17.4\% of cases). Stillborn neonates had a high incidence of chorioamnionitis $(40 \%$ vs. $16 \%$ ), funisitis $(40 \%$ vs. $8 \%$ ) and villitis $(40 \%$ vs. $8 \%)$ in their placenta. Significant changes were found in the placentas of the neonates who expired in their neonatal period. Placentas in low birth weight babies were found to have marginally higher chorioamnionitis $(22 \%$ vs. $16 \%)$, increased villitis (22\% vs. $8 \%)$, syncytial knotting (37\% vs. 16\%), and cytotrophoblast proliferation $(22 \%$ vs. $8 \%)$. Conclusion: The inflammatory lesions were found to be highly prevalent in the stillborn highlighting a significant role of the infection in the causation of preterm delivery and subsequently in neonatal morbidity and mortality. So, placental pathological analysis is very important to predict the risk of developing serious inflammatory complications in neonates.
\end{abstract}

Keywords: Placenta, Neonatal outcome, Pregnancy, Histopathology

\section{Introduction}

The placenta is a vital organ responsible for gaseous and nutrition transfer from mother to the fetus as well as disposable of waste products of the fetus. It is a metabolic and endocrine organ of supreme importance for establishment and maintenance of the pregnancy. Origin of the term placenta is controversial. Some give this credit to Gabriele de Falloppio (1532-1562), after whom fallopian tubes are named. While some believe that the term "Placenta" was introduced by Realdus Columbus (1559) and was derived from the Latin word "Palkus" meaning circular/flat cake. "Secunidinae" was another popular name for it in early days [1].

Since it is the only point of contact between maternal and fetal tissues, anything which affects the fetus can do so only through the placenta. It is also important for immunological acceptance of the fetus by the mother [1]. Any maternal diseases, acute or chronic, have its

Manuscript received: $8^{\text {th }}$ August 2018

Reviewed: $18^{\text {th }}$ August 2018

Author Corrected: $25^{\text {th }}$ August 2018

Accepted for Publication: $30^{\text {th }}$ August 2018 effect on fetus by altering the placental metabolism and transfer mechanism. Any such compromise is likely to leave its impression on the placenta, which can be detected by pathological and ultrasonographic examinations. But one should not be over expecting from placental examinations since placental change are a manifestation of primary compromise rather than the primary cause of neonatal morbidity [2].

Neonatal morbidity and mortality may have its root in the placenta which we can be studied through the pathological examination. Although placental changes do not have a highly specific picture, they do give an indication of the pathophysiological process involved. Previous workers have found placental pathological changes associated with different morbidities like neonatal septsis $[3,4]$, prematurity $[5,6,7]$, Intrauterine growth restriction (IUGR) [7,8,9], birth asphyxia $[10,11]$, stillbirth [12,13]. Placental changes show a significant association with neonatal morbidity and mortality and placental examination offers a lot of prognostic significance for the newborn [14]. Hence, 


\section{Original Research Article}

this study was designed to explore the correlation of placental histopathology with neonatal outcome.

\section{Material and Methods}

A study aimed at finding the association between the histopathological changes in placenta and outcome in the neonate was conducted at the Department of Obstetrics and Gynecology (with newborns admitted at Department of Pediatrics) and histopathological examination was done in the Department of Pathology of a Medical College of Raipur.

Ethical approval: The study protocol was reviewed by the Ethical committee of the Institutional Review Board and was granted ethical clearance. Written informed consent was taken from the subject or their family members.

Study subjects: In the present study, 100 placentas (with their membrane and cords) were included. Among them, 76 placentas belonging to neonates with different morbidities like prematurity (20 cases), IUGR (23 cases), birth asphyxia (44 cases), low birth weight (27 cases), septicemia (15 cases), perinatal mortalities viz. still birth (10 cases) and neonatal death (6 cases). These morbidities were studied for histopathological changes.

Inclusion criteria: The following inclusion criteria were used:

$>$ Preterm: Neonate delivering before the 37 completed weeks, (gestation age calculated by LMP and physical examination).

$>$ IUGR: Defined as birth weight $<10$ th centile for age.

> Birth asphyxia: APGAR (Appearance, Pulse, Grimace, Activity, Respiration) scores of $<7$ at 1 $\min$.

$>$ Still Born: Neonate showing no sign of life after delivery, including intermediate (22-27weeks) and late ( $>28$ weeks) fetal death.

$>$ Low birth weight: Babies with a birth weight of $2500 \mathrm{~g}$ irrespective of the period of gestation.

$>$ Septicemia: Isolation of pathogens either from blood, cerebrospinal fluid (CSF), urine or trachea.
Sampling technique: Convenient sampling was used. Neonates fulfilling the inclusion criteria were included in the study.

Data Collection: From neonates, Apgar score at $1 \mathrm{~min}$. and resuscitation events were noted. Birth weight was noted and gestation age was determined using the New Ballard score.

Using population data, it was determined whether birth weight was appropriate for gestation age or not and thus the baby was labeled AGA (Appropriate for gestational age) or IUGR.

General examination: General and systemic examination were done. Babies were followed up till discharge of the mother and baby (if admitted at the nursery) for any morbidity or mortality.

Examination of the placenta: Placentas were collected from mothers delivering at the Hospital. After collecting, the placenta was examined for gross findings such as infraction or calcification, retroplacental clots and infraction. Then they were fixed in $10 \%$ formalin for 48 hours. After 48 hours of fixation in formalin, 6 strips of $0.5 \mathrm{~cm}$ were taken from cord, central portion (1 fetal and 1 maternal), peripheral portion ( 1 fetal and 1 maternal), any gross lesion or representative area.

After embedding in paraffin wax sections of 5-7 micrometer were taken with the microtome. Sections were stained with Hematoxylin and Eosin stain. Placenta, cord and membrane were examined for the following pathological changes: chorioamnionitis, infarction, villitis, funisitis, syncytial knots, fibrinoid necrosis, cytotrophoblast proliferation, villous stromal fibrosis, calcification and umbilical thrombosis

Statistical analysis: Data thus collected, was analyzed using the chi-square test $\left(\chi^{2}\right.$ test) for the significance of the difference in the proportion of pathological change in various groups studied. The test was applied and pvalue was calculated using MS Excel 2003, Data analysis tool. p-value $<0.05$ was considered as statistically significant.

\section{Results}

In our study, a higher incidence of inflammatory lesions in the form of chorioamnionitis (30\%) was found in preterm neonates (PT) as compared to healthy full terms newborn (FT). Incidence of infarction (60\% in PT vs. $45 \%$ in FT), villitis (20\% vs. $8 \%$ ) fibrinoid necrosis (90\% vs.66\%), cytotrophoblast proliferation (20\% vs. $10 \%)$, and villous stromal fibrosis $(90 \%$ vs. $81 \%$ ) was also increased, while incidence of calcification was found to be reduced (20\% vs. $36 \%)$.

Most of the results were nearly approaching statistical significance but statistical significance was found only with cytotrophoblast proliferation (p-0.02). (Table1) 


\section{Original Research Article}

Table-1: Comparison of the placental pathology of preterm and fullterm neonates without risk factors.

\begin{tabular}{|c|c|c|c|c|c|}
\hline Pathology & \multicolumn{2}{|c|}{ Preterm (20) } & \multicolumn{2}{|c|}{ Healthy Full-term (24) } & p- value \\
\hline Chorioamnionitis & 6 & $30.0 \%$ & 4 & $16.7 \%$ & 0.29 \\
\hline Infarction & 12 & $60.0 \%$ & 11 & $45.8 \%$ & 0.35 \\
\hline Villitis & 4 & $20.0 \%$ & 2 & $8.3 \%$ & 0.26 \\
\hline Funisits: & 0 & $0.0 \%$ & 2 & $8.3 \%$ & 0.19 \\
\hline Syncytial knots & 6 & $30.0 \%$ & 4 & $16.7 \%$ & 0.29 \\
\hline Fibrinoid Necrosis & 18 & $90.0 \%$ & 16 & $66.7 \%$ & 0.07 \\
\hline Cytotrophoblast proliferation & 4 & $20.0 \%$ & 0 & $0.0 \%$ & $* \mathbf{0 . 0 2}$ \\
\hline Villous stromal Fibrosis & 18 & $90.0 \%$ & 16 & $66.7 \%$ & 0.07 \\
\hline Calcification & 4 & $20.0 \%$ & 9 & $37.5 \%$ & 0.21 \\
\hline Umbilical thrombotic vasculopathy & 2 & $10.0 \%$ & 2 & $8.3 \%$ & 0.85 \\
\hline
\end{tabular}

* denotes statistical significance $(\mathbf{p}<0.05)$

Among 20 preterm, 12 babies were without preeclamptic history (Non PE) and 8 were with preeclamptic history (PE). On comparison, chorioamnionitis (33\% in Non PEPT vs. $25 \%$ in PEPT) followed by syncytial knots (33\% vs. $25 \%)$ were found to be increased in non PE group while PE group terms had more of infarction (45\% vs. 34\%) and fibrinoid necrosis $(100 \%$ vs. $83 \%)$. Incidence of infarction was found statistically significant higher in preeclamptic (Table 2).

Table-2: Comparison of the placental pathology of Non-Pre-eclamptic preterm and Pre-eclamptic preterm neonates without risk factors.

\begin{tabular}{|c|c|c|c|c|c|}
\hline Pathology & \multicolumn{2}{|c|}{ Non PE Preterms (12) } & \multicolumn{2}{|c|}{ PE Preterms $(\mathbf{8})$} & p- value \\
\hline Chorioamnionitis & 4 & $33.3 \%$ & 2 & $25.0 \%$ & 0.69 \\
\hline Infarction & 4 & $33.3 \%$ & 8 & $100.0 \%$ & $* \mathbf{0 . 0 0 2 9}$ \\
\hline Villitis & 2 & $16.7 \%$ & 2 & $25.0 \%$ & 0.65 \\
\hline Funisits & 0 & $0.0 \%$ & 0 & $0.0 \%$ & 0.65 \\
\hline Syncytial knots & 4 & $33.3 \%$ & 2 & $25.0 \%$ & -- \\
\hline Fibrinoid Necrosis & 10 & $83.3 \%$ & 8 & $100.0 \%$ & 0.22 \\
\hline Cytotrophoblast proliferation & 2 & $16.7 \%$ & 2 & $25.0 \%$ & 0.65 \\
\hline Villous stromal Fibrosis & 10 & $83.3 \%$ & 8 & $100.0 \%$ & 0.22 \\
\hline Calcification & 2 & $16.7 \%$ & 2 & $25.0 \%$ & 0.65 \\
\hline Umbilical thrombotic vasculopathy & 2 & $16.7 \%$ & 0 & $0.0 \%$ & 0.22 \\
\hline
\end{tabular}

* denotes statistical significance $(\mathbf{p}<0.05)$

In our study, a higher incidence of villitis was found in IUGR neonates (17.4\% of cases). A higher incidence of calcification was also found although it does not reach statistical significance. Infarction and cytotrophoblast proliferation were also found to be increased $(64 \%, 8.7 \%)$ in IUGR babies (Table 3$)$.

Table-3: Placental pathological changes in IUGR pregnancies.

\begin{tabular}{|c|c|c|c|c|c|}
\hline Pathology & \multicolumn{2}{|c|}{ IUGR (23) } & \multicolumn{2}{c|}{ Healthy AGA } & p- value \\
\hline Chorioamnionitis & 2 & $8.7 \%$ & 4 & $16.7 \%$ & 0.41 \\
\hline Infarction & 8 & $34.8 \%$ & 11 & $45.8 \%$ & 0.44 \\
\hline Villitis & 4 & $17.4 \%$ & 2 & $8.3 \%$ & 0.35 \\
\hline Funisits & 0 & $0.0 \%$ & 2 & $8.3 \%$ & 0.16 \\
\hline Syncytial knots & 8 & $34.8 \%$ & 4 & $16.7 \%$ & 0.15 \\
\hline Fibrinoid Necrosis & 14 & $60.9 \%$ & 16 & $66.7 \%$ & 0.68 \\
\hline Cytotrophoblast proliferation & 2 & $8.7 \%$ & 0 & $0.0 \%$ & 0.14 \\
\hline Villous stromal Fibrosis & 21 & $91.3 \%$ & 16 & $66.7 \%$ & $* \mathbf{0 . 0 4}$ \\
\hline Calcification & 10 & $43.5 \%$ & 9 & $37.5 \%$ & 0.68 \\
\hline Umbilical thrombotic vasculopathy & 2 & $8.7 \%$ & 2 & $8.3 \%$ & 0.96 \\
\hline
\end{tabular}

* denotes statistical significance $(\mathbf{p}<0.05)$ 


\section{Original Research Article}

There was no significant increase in the inflammatory lesions in the form of chorioamnionitis in cases of birth asphyxia (18\% vs. $16 \%)$, although villitis does show some increase (18.2\% in birth asphyxia vs. $8.3 \%$ in healthy newborns without any maternal risk factors, not significant statistically) (Table 4).

Table-4: Placental histopathological changes in birth asphyxia neonates.

\begin{tabular}{|c|c|c|c|c|c|}
\hline Pathology & \multicolumn{2}{|c|}{ IUGR (23) } & \multicolumn{2}{|c|}{ Healthy AGA } & p- value \\
\hline Chorioamnionitis & 8 & $18.2 \%$ & 4 & $16.7 \%$ & 0.87 \\
\hline Infarction & 24 & $54.5 \%$ & 11 & $45.8 \%$ & 0.49 \\
\hline Villitis & 8 & $18.2 \%$ & 2 & $8.3 \%$ & 0.27 \\
\hline Funisits & 0 & $0.0 \%$ & 2 & $8.3 \%$ & $* \mathbf{0 . 0 5}$ \\
\hline Syncytial knots & 20 & $45.5 \%$ & 4 & $16.7 \%$ & $* \mathbf{0 . 0 2}$ \\
\hline Fibrinoid Necrosis & 26 & $59.1 \%$ & 16 & $66.7 \%$ & 0.54 \\
\hline Cytotrophoblast proliferation & 8 & $18.2 \%$ & 0 & $0.0 \%$ & $* \mathbf{0 . 0 3}$ \\
\hline Villous stromal Fibrosis & 40 & $90.9 \%$ & 16 & $66.7 \%$ & $* \mathbf{0 . 0 1}$ \\
\hline Calcification & 18 & $40.9 \%$ & 9 & $37.5 \%$ & 0.78 \\
\hline Umbilical thrombotic vasculopathy & 2 & $4.5 \%$ & 2 & $8.3 \%$ & 0.53 \\
\hline
\end{tabular}

* denotes statistical significance $(\mathbf{p}<\mathbf{0 . 0 5})$

Still born neonates had a high incidence of chorioamnionitis ( $40 \%$ vs. $16 \%$ ), funisitis ( $40 \%$ vs. $8 \%$ ) and villitis ( $40 \%$ vs. $8 \%)$ in their placenta. Very high incidence of infarction ( $80 \%$ vs. $45 \%)$ was also found (Table 5).

Table-5: Placental pathological changes in stillbirths.

\begin{tabular}{|c|c|c|c|c|c|}
\hline Pathology & \multicolumn{2}{|c|}{ Still birth (10) } & \multicolumn{2}{|c|}{ Healthy (24) } & p- value \\
\hline Chorioamnionitis & 4 & $40.0 \%$ & 4 & $16.7 \%$ & 0.14 \\
\hline Infarction & 8 & $80.0 \%$ & 11 & $45.8 \%$ & 0.07 \\
\hline Villitis & 4 & $40.0 \%$ & 2 & $8.3 \%$ & $* \mathbf{0 . 0 3}$ \\
\hline Funisits & 4 & $40.0 \%$ & 2 & $8.3 \%$ & $* \mathbf{0 . 0 3}$ \\
\hline Syncytial knots & 6 & $60.0 \%$ & 4 & $16.7 \%$ & $* \mathbf{0 . 0 1}$ \\
\hline Fibrinoid Necrosis & 8 & $80.0 \%$ & 16 & $66.7 \%$ & 0.44 \\
\hline Cytotrophoblast proliferation & 2 & $20.0 \%$ & 0 & $0.0 \%$ & $* \mathbf{0 . 0 2}$ \\
\hline Villous stromal Fibrosis & 8 & $80.0 \%$ & 16 & $66.7 \%$ & 0.44 \\
\hline Calcification & 4 & $40.0 \%$ & 9 & $37.5 \%$ & 0.89 \\
\hline Umbilical thrombotic vasculopathy & 0 & $0.0 \%$ & 2 & $8.3 \%$ & 0.35 \\
\hline
\end{tabular}

* denotes statistical significance $(\mathbf{p}<0.05)$

Significant changes were found in the placentas of the neonates who expired in their neonatal period. A higher incidence of chorioamnionitis (33.3\% vs. $15 \%$ ), infarction (all 6 placentas vs. $45 \%$ ), villitis (33\% vs. $12 \%$ ) and villous stromal fibrosis $(100 \%$ vs. $82 \%)$ was found. Incidence of calcification was same.

Table-6: Placental Pathology in neonatal deaths.

\begin{tabular}{|c|c|c|c|c|c|}
\hline Pathology & \multicolumn{2}{|c|}{ Neonatal Deaths (10) } & \multicolumn{2}{|c|}{ Healthy (24) } & p- value \\
\hline Chorioamnionitis & 2 & $20.0 \%$ & 4 & $16.7 \%$ & 0.14 \\
\hline Infarction & 6 & $60.0 \%$ & 11 & $45.8 \%$ & 0.07 \\
\hline Villitis & 2 & $20.0 \%$ & 2 & $8.3 \%$ & $* \mathbf{0 . 0 3}$ \\
\hline Funisits & 0 & $0.0 \%$ & 2 & $8.3 \%$ & $* \mathbf{0 . 0 3}$ \\
\hline Syncytial knots & 2 & $20.0 \%$ & 4 & $16.7 \%$ & $* \mathbf{0 . 0 1}$ \\
\hline Fibrinoid Necrosis & 4 & $40.0 \%$ & 16 & $66.7 \%$ & 0.44 \\
\hline Cytotrophoblast proliferation & 0 & $0.0 \%$ & 0 & $0.0 \%$ & $* \mathbf{0 . 0 2}$ \\
\hline Villous stromal Fibrosis & 6 & $60.0 \%$ & 16 & $66.7 \%$ & 0.44 \\
\hline Calcification & 2 & $20.0 \%$ & 9 & $37.5 \%$ & 0.89 \\
\hline Umbilical thrombotic vasculopathy & 0 & $0.0 \%$ & 2 & $8.3 \%$ & 0.35 \\
\hline
\end{tabular}

* denotes statistical significance $(\mathbf{p}<\mathbf{0 . 0 5})$ 


\section{Original Research Article}

Placentas in low birth weight babies was found to have marginally higher chorioamnionitis ( $22 \%$ vs. $16 \%)$, increased villitis $(22 \%$ vs. $8 \%)$, syncytial knotting ( $37 \%$ vs. $16 \%)$, and cytotrophoblast proliferation $(22 \%$ vs. $8 \%)$. Among these factors, cytotrophoblast proliferation ( $\mathrm{p}-0.01)$ and villous stromal fibrosis ( $\mathrm{p}-0.02)$ was statistically significant.

A very high incidence of infective and inflammatory lesions in the form of chorioamnionitis ( $60 \%$ vs. $10 \%)$ and villits ( $26 \%$ vs. $14 \%$ ) was found in neonates with septicemia. There was no significant increase in any other pathology except villous stromal fibrosis $(86 \%$ vs. $66 \%$ ) in neonates with septicemia. Chorioamnionitis (p-0.01) and umbilical thrombotic vasculopathy (p-0.04) was statistically significant.

\section{Discussion}

In the present study, preterm neonates were found to have more of infective and inflammatory lesions in the form of chorioamnionitis, indicating the role of infection in the causation of preterm delivery and subsequent morbidity. Higher incidence of the placental inflammatory lesion in the form of chorioamnionitis was found by many workers in the past. Geoffrey Altshuler [15] also found a significant association between prematurity and chorioamnionitis (43\% in preterm while $18 \%$ in full term). F Arias [16] found inflammatory lesions in $45 \%$ and vascular lesions (infarction) in $40 \%$ preterms. Robert W. Novak [3] and Ogunyemi D et al [6] also found a significant association between chorioamnionitis and preterm delivery. The excess of inflammatory/infective changes in the form of chorioamnionitis and villitis in the placentas of preterm further substantiates the importance of infection and hence inflammation in the pathophysiology of causation of preterms. The mechanism proposed is mainly elaboration of cytokines including prostaglandins, which on one hand increase myometrial contractions and on the other hand increases metalloproteases, which in turn induce chorioamnion weakening, rupture and also induce cervical ripening, all culminating into preterm delivery [15].

High incidence of maternal vasculopathy was found in the present study as well as in the previous studies. Fernando A et al[16] also found maternal vasculopathy in the form of infarction. Inadequate maternal blood flow due to inadequate conversion of the spiral arteries is the proposed cause of fetal compromise according to him.

In the present study, chorioamnionitis, syncytial knots were found to be increased in non-PE group while PE had more of infarction, fibrinoid necrosis, cytotrophoblastic proliferation, and villous stromal fibrosis. Carolyn M et al (1995)[17] in their comparison also had more or less or similar finding with more of acute inflammatory lesions like chorioamnionitis in spontaneous prematurity group than preeclamptic group (44\% vs. $20 \%)$ and increased vascular lesions like cytotrophoblast hyperplasia ( $42 \%$ vs. $14 \%$ ), villous fibrosis ( $83 \%$ vs. $33 \%$ ) and infarction $(45 \%$ vs. $22 \%)$ in the preeclamptic group. This pattern of distribution with preterm eclamptic having less of an inflammatory lesion and more of vascular lesion indicate a difference in pathophysiology of the two groups and that immunopathologic processes and coagulation may be involved in the pathophysiological mechanisms of preterm preeclampsia, independent of uteroplacental vascular pathologic features.

A higher incidence of villitis was found in IUGR neonates, which was in agreement with previous studies. Anjali R Madoskar et al[18] found increased syncytial knotting in 30\%, fibrinoid necrosis in 30\%, increased basement membrane thickness in $90 \%$, cytotrophoblastic hyperplasia in $70 \%$ and villous fibrosis in 30\% of IUGR cases. Redfine RW[7] found an association between villitis and IUGR. David M Becroft [9] found a positive association between villitis of unknown etiology and an incidence of SGA (Small for gestational age) (17.3\% vs.11.7\% in AGA). Villitis is considered to have an immunological basis and immunological injury may be due to an initial infective (most likely virus) insult. It is considered to hamper the feto-maternal transfer of nutrients and is known to be associated with IUGR.

In the current study, chorioamnionitis was found to be similar/ slightly increased, while villitis is found to be increased. Raymond et al [19] found chorioamnionitis in $26 \%$ of full-term birth asphyxia cases as compared to $20 \%$ in controls. Rasiah Vegnewaran et al [20] found no significant difference in inflammatory changes in the form of and birth asphyxia (41\% vs. $37 \%$ ). Decreased incidence of inflammatory changes in the current study is due to the fact most of the birth asphyxia cases in the study were full-term. The study underlines the importance of infective lesions in the form of chorioamnionitis in the low birth weight (LBW) infants (by increasing preterm deliveries).

Ogunyemi D et al[6] also found a significant association between infarction and LBW. The low birth weight of baby may be due to two reasons, preterm or an IUGR baby. Both have different pathophysiological significance. When discussed together, a baby born with weight $<2.5 \mathrm{~kg}$ is more likely to have marginally higher chorioamnionitis. 


\section{Original Research Article}

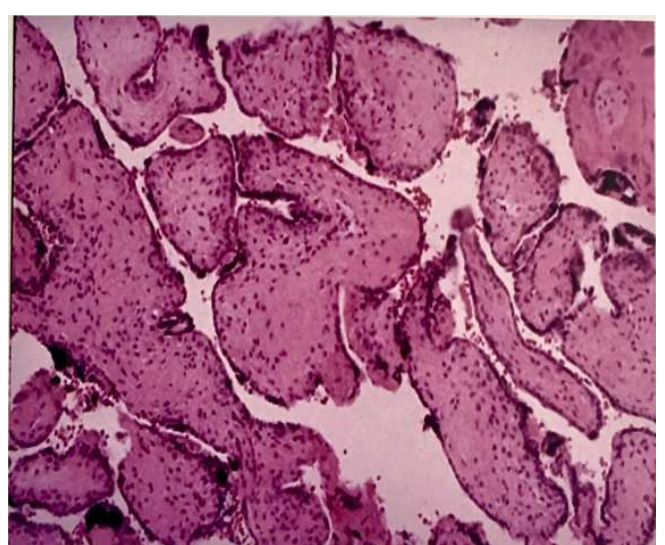

Figure 1: Photomicrograph of placenta showing villous fibrosis.

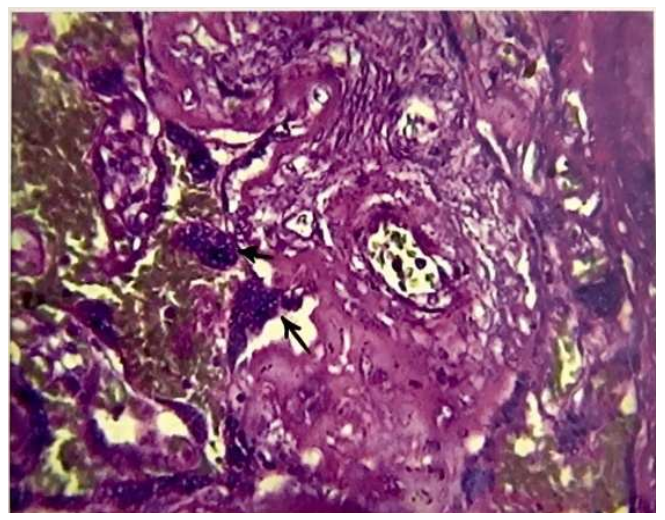

Figure 3: Photomicropgraph of placenta showing syncytial knotting.

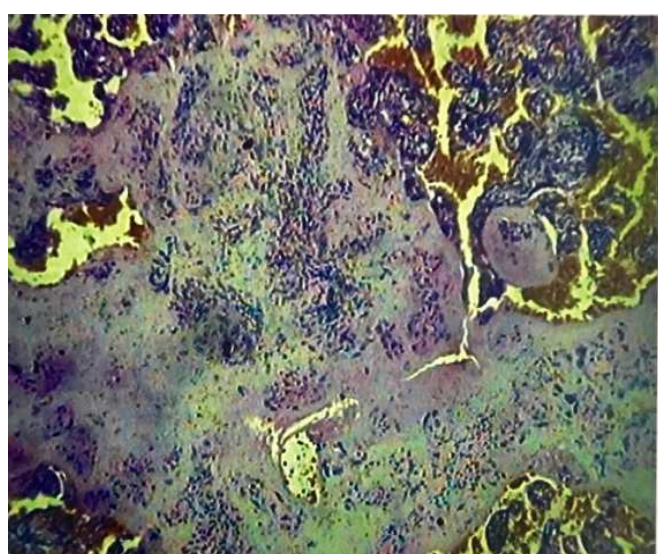

Figure 5: Photomicropgraph of placenta showing infarction.

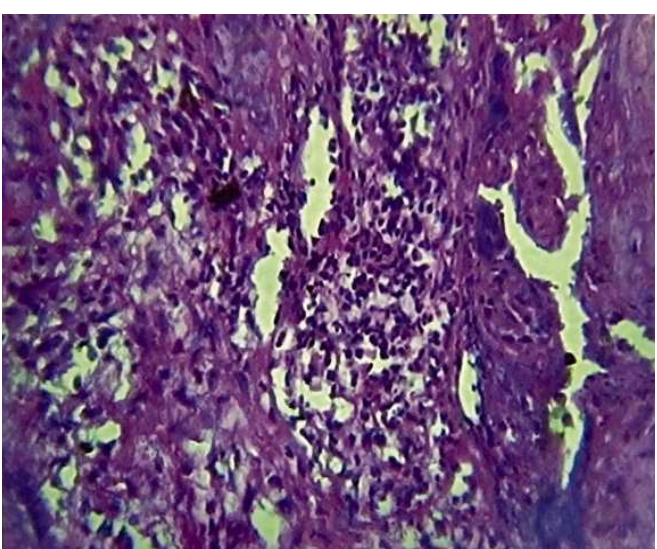

Figure 2 : photomicrograph of placenta showing villitis.

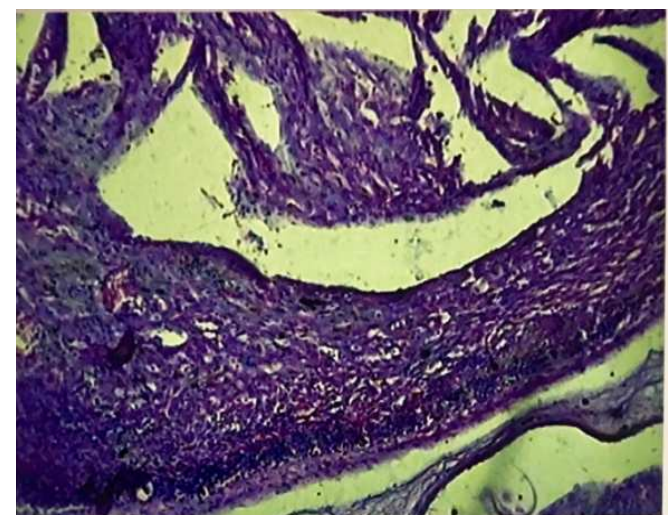

Figure 4: Photomicropgraph of placenta showing chorioamnionitis.

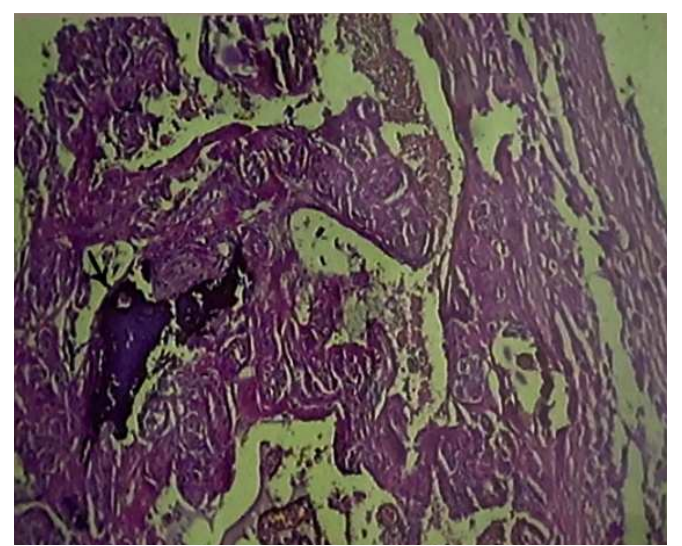

Figure 6: Photomicropgraph of placenta showing calcification.

A very high incidence of infective and inflammatory lesions in the form of chorioamnionitis and villits was found in neonates with septicemia. Rajiv Mehta et al [21] and Robert W. Novak [3] also found chorioamnionitis in about 70\% cases and $64 \%$ cases of septicaemia respectively. Ogunyemi D et al (2003)[6]found a significant association between chorioamnionitis and neonatal sepsis.

Stillborn neonates had a high incidence of chorioamnionitis, funisitis and villitis. Harvey J Kliman, [22] found evidence of placental infection in 17-33\% of stillbirths. Ogunyemi D et al[6], Geoffrey et al [15] found a significant association between chorioamnionitis and neonatal mortality, indicating the role of infection and mortality. Richard L Naeye [23] found placental involvement in a large proportion of perinatal mortality. He found that $17 \%$ of deaths were due to 


\section{Original Research Article}

amniotic fluid infections, and 6\% to large placental infarcts. Eumenia Costa et al (2004)[24] have also found a significant association between villitis and stillbirth as in the current study. Stillborn showed more severe inflammatory/infective lesions in the form of funisitis. Other changes in the stillbirth were secondary to fetal death either in the form degenerative change (infarction, villous fibrosis) due to reduced maternal and fetal blood flow or in the form of reparative hyperplasic response as placenta depends on the maternal supply for its survival and remains very much viable after fetal death.

\section{Conclusion}

Preterm neonates were found to have more of infective and inflammatory lesions in the form of chorioamnionitis, villitis, funisitis, IUGR etc. These lesions were highly prevalent in the stillborn highlighting a significant role of the infection in the causation of preterm delivery and subsequently in neonatal morbidity and mortality.

Hence, during pregnancy identification of these inflammatory lesions should be done using some markers of inflammatory status for an early diagnosis and a detailed monitoring of pregnancy course.

Placental pathological analysis is very important to predict the risk of developing serious inflammatory complications in neonates.

What this study adds to existing knowledge- The placental histology plays a key role in determining the fetal and neonatal mortality, morbidity, and outcome. So, Pediatricians and Gynecologists should try to obtain the results of histopathological examination of placenta.

Funding: Nil, Conflict of interest: None initiated Permission from IRB: Yes

\section{References}

1. Norwitz ER, Schust DJ, Fisher SJ. Implantation and the survival of early pregnancy. DOI:10.1056/ NEJMra 000763

2. Fox H. The histopathology of placental insufficiency. J Clin Pathol Suppl (R Coll Pathol). 1976;10:1-8.

3. Novak RW, Platt MS. Significance of placental findings in early-onset group B streptococcal neonatal sepsis. DOI:10.1177/000992288502400504

4. Kim CJ, Romero R, Chaemsaithong $\mathrm{P}$, et al. Acute chorioamnionitis and funisitis: definition, pathologic features, and clinical significance. DOI:10.1016/j.ajog. 2015. 08.040

5. Arias F, Victoria A, Cho K, Kraus F. Placental histology and clinical characteristics of patients with preterm premature rupture of membranes. DOI:10. 1016/ S0029-7844(96)00451-6

6. Ogunyemi D, Murillo M, Jackson U, et al. The relationship between placental histopathology findings and perinatal outcome in preterm infants. DOI:10. 1080/ jmf. 13.2.102.109

7. Redline RW, Patterson P. Patterns of placental injury. Correlations with gestationalage, placental weight and clinical diagnoses. Arch Pathol Lab Med. 1994 Jul; 118 (7): 698-701.

8. Altshuler G, Russell P, Ermocilla R. The placental pathology of small-for-gestational age infants. Am J Obstet Gynecol. 1975 Feb 1; 121 (3) : 351-9.

9. Becroft DM, Thompson JM, Mitchell EA. Placental villitis of unknown origin: epidemiologic associations.

DOI:10.1016/j.ajog.2004.06.062

10. Redline RW, Wilson-Costello D, Borawski E, et al. Placental lesions associated with neurologic impairment and cerebral palsy in very low-birth-weight infants. Arch Pathol Lab Med. 1998 Dec; 122 (12): $1091-8$.

11. Redline RW, O'Riordan MA. Placental lesions associated with cerebral palsy and neurologic impairment following term birth. DOI:10. 1043/00039985 (2000)124<1785: PLAWCP>2.0.CO;2

12. Ornoy A, Crone K, Altshuler G. Pathological features of the placenta in fetal death. Arch Pathol Lab Med. 1976 Jul;100(7):367-71.

13. Pinar H, Goldenberg RL, Koch MA, Placental findings in singleton stillbirths. DOI:10.1097/AOG. 0000000000000100

14. Aladjem S, Perrin E, Fanaroff A. Placental score and neonatal outcome: a clinical and pathologic study. Obstet Gynecol. 1972 Apr; 39 (4) : 591602.

15. Altshuler G, Hyde S. Clinicopathologic considerations of fusobacteria chorioamnionitis. Acta Obstet Gynecol Scand. 1988;67(6): 513-7. 


\section{Original Research Article}

16. Arias F, Victoria A, Cho K, Kraus F. Placental histology and clinical characteristics of patients with preterm premature rupture of membranes. DOI:10. 1016/ S0029-7844(96)00451-6

17. Salafia CM, Minior VK, López-Zeno JA, et al. Relationship between placental histologic features and umbilical cord blood gases in preterm gestations. Am J Obstet Gynecol. 1995 Oct;173(4):1058-64.

18. Anjali R. Masodkar. Histopathology of placenta and its correlation: worth outcome: Journal of Obstetrics and Gynaecology of India, 1985; 35 (2): 294300 .

19. Redline RW, O'Riordan MA. Placental lesions associated with cerebral palsy and neurologic impairment following term birth. DOI:10.1043/00039985 (2000)124<1785:PLAWCP>2.0.CO;2
20. RasiahVigneswaran et al. Cerebral palsy and placental infection. BMC pregnancy and child birth. 2004; 4:1:28-32

21. Mehta Rajeev, Nanjundaswamy Shakuntala, ShenSchwarz Susan, Petrova Anna et al. Neonatal morbidity and placental pathology. Indian Journal of Pediatrics. 2006;73 (1): 25-28

22. Kliman HJ. Uteroplacental blood flow. The story of decidualization, menstruation, and trophoblast invasion. Am J Pathol. 2000 Dec;157(6):1759-68.

23. Naeye RL. Causes of perinatal mortality in the US Collaborative Perinatal Project. JAMA. 1977 Jul 18; 238 (3): 228-9.

24. Eumenia Costa. Placental vlllitis: Fetal and maternal outcome.Rev.Bras. Ginecol Obstet. 2004;26(10):807-12

\section{How to cite this article?}

Kumar S, Sudarshan V. The correlation of placental histopathology with neonatal outcome. Trop J Path Micro 2018; 4(4):336-343.doi:10. 17511/ jopm. 2018.i4.07 